\title{
La radiographie mobile: assistance à notre population vieillissante et à mobilité réduite?
}

\author{
Pour les patients âgés ou handicapés, un examen radiologique est souvent associé \\ à des coûts considérables. Rien que le transport, éventuellement accompagné d'une \\ personne, est déjà compliqué et cher. La radiographie ambulatoire peut apporter \\ une solution.
}

Forat Sadry, Andreas

Nidecker, Jean-Charles Piguet, Bernhard Burckhardt
Liens d'intérêts: Les auteurs sont cofondateurs de MOBIRAD Sàrl, créée à titre d'essai pilote, tant dans les cantons de Bâle-Ville et Bâle-Campagne que dans le canton de Genève, afin de démontrer l'utilité et le besoin de la radiologie mobile.

Correspondance: Dr Forat Sadry Présidente MOBIRAD Sàrl c/o Givision imagerie diagnostique Rte. A. Piller 29 CH-1762 Givisiez

f.sadry@givision.ch
La réalisation d'un examen diagnostique radiologique pour une personne âgée et/ou à mobilité réduite peut s'avérer un vrai casse-tête: il faut organiser son déplacement au service de radiologie et prévoir très souvent du personnel accompagnant. Le cas échéant, c'est à l'ambulance qu'il faut recourir avec le coût que cela représente. Sur place, le temps d'attente est variable et l'exécution de l'examen s'avère compliquée et parfois désagréable pour la personne (déshabillage, positionnement, attente pendant la réalisation des images). Enfin, le patient est très souvent renvoyé à domicile après exécution des radiographies.

Tous ces facteurs expliquent l'hésitation à envoyer ces patients pour un examen diagnostique.

En 2005, le Professeur Frode Laerum à Oslo proposait une solution quant au type de soins qui pouvaient être prodigués à une population à mobilité réduite [1]. Tenant compte d'une population vieillissante et de l'augmentation du nombre de personnes handicapées [2, 3], notamment en institution, il a eu l'idée de faire profiter cette part de la population de la technologie radiologique moderne. Il a réalisé le projet d'offrir la possibilité d'être radiographié au domicile ou au lieu du séjour du patient. Nous avons décidé d'offrir ce service à titre d'essai en Suisse. Notre cible était prioritairement les maisons de retraite et établissements médico-sociaux.

La radiologie mobile est déjà employée dans les hôpitaux depuis de longues années, notamment dans les salles d'opération et dans les services d'urgence. Ces appareils radiologiques sont cependant très encombrants et pèsent souvent plus de $500 \mathrm{~kg}$. Comme dans beaucoup de domaines, grâce aux progrès technologiques considérables, les appareils radiologiques sont également devenus plus petits et plus performants tout en minimisant les doses de rayons $\mathrm{X}$ émises: il est possible de nos jours d'employer du matériel mobile ne pesant pas plus de $80 \mathrm{~kg}$ et d'assurer l'accès très rapide aux images par un radiologue travaillant à distance. Les clichés peuvent être imprimés sur film ou sur papier, mais également gravés sur CD ou sauvegardés sur une clé USB.

Le projet MOBIRAD a débuté en mars 2007 dans les Cantons de Bâle-Ville et Bâle-Campagne, puis à
Genève en mai 2007 [4, 5]. Malgré des efforts considérables, le projet à Bâle a du être abandonné après plusieurs mois en raison d'un défaut d'infrastructure de soutien. Les frais engendrés ne permettaient pas de poursuivre l'expérience au grand regret des quelques médecins référents qui ont su profiter d'un service rendu avec rapidité.

A Genève, l'expérience a été tout autre: Evitant quelques-uns des écueils des débuts Bâlois et soutenus par toute une équipe, un radiologue et un technicien en radiologie ont réussi à démontrer l'utilité publique et le besoin de cette radiologie ambulatoire.

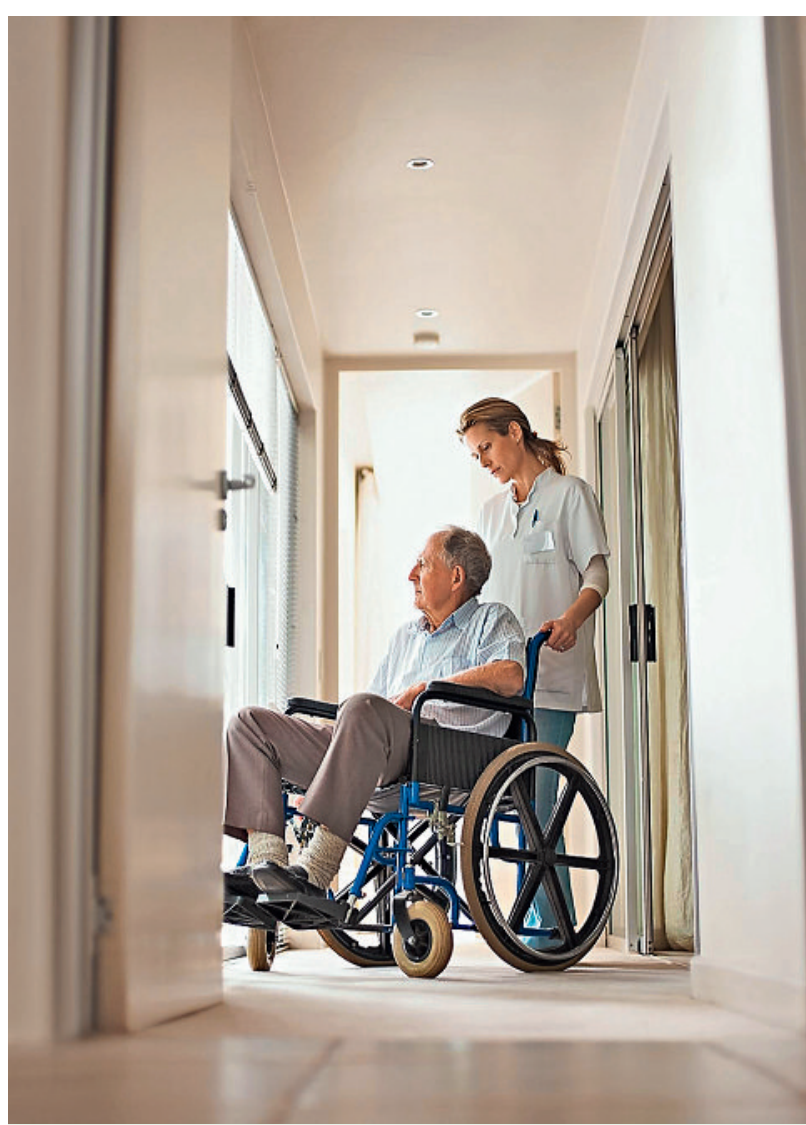

Emmener des personnes âgées ou handicapées à leurs examens radiologiques requiert souvent du temps et de l'argent. 


\section{Premier résultats du projet}

Pour étayer nos hypothèses, tous les établissements qui ont profité de notre service et tous les médecins référents ont été soumis à un questionnaire pendant la première année d'exploitation (2007). Ce questionnaire s'adressait au médecin prescripteur et à l'équipe soignante avec comme but l'évaluation de la satisfaction quant aux services rendues: moyen de transport utilisé? Avec ou sans accompagnant? Si les circonstances en changeaient l'indication? Le type d'examen et nombre de radiographies effectuées ... Le taux de réponse a été plus important à Bâle où moins d'examens ont été effectués qu'à Genève où les médecins trouvaient fastidieux de remplir le questionnaire à chaque demande.

A Bâle, il y a eu 70 patients répartis dans 30 EMS en une année: les données ont été remplies pour 57/70 patients: le médecin a exprimé sa satisfaction dans $90 \%$. Dans $93 \%$ (53/57 cas), l'examen aurait été prescrit de toute façon. Le transport était à prévoir dans tous ces cas et l'accompagnement dans 56/57 (98\%). Dans 7 cas, un taxi aurait été utilisé et dans 50 cas une ambulance.

A Genève, il y a eu 261 patients en six mois: le taux de retour du questionnaire a été de $33 \%$. L'indice de satisfaction médicale était de $9 / 10$ et 10/10 dans $82 \%$ des cas. Dans $94,6 \%$ des cas $(246 / 261)$, l'examen aurait été prescrit de toute façon avec le besoin d'un transport dans chaque cas et d'un accompagnant dans 204/261 des cas (77\%). Parmi les réponses données, un taxi ou un véhicule privé aurait été utilisé dans 60 cas et dans 56 cas une ambulance.

En deux ans, plus de 1800 personnes ont pu bénéficier de ce service: les indications posées étaient des recherches de fracture après chute ou l'étiologie de douleurs handicapantes ou encore la confirmation d'une décompensation cardiaque ou une infection pulmonaire. Dans la majorité des cas, les transports aller-retour vers des cabinets de radiologie ou des hôpitaux ont pu être évités, même si certaines situations ont nécessité l'hospitalisation.

Si le concept d'une radiologie «à domicile» peut être considéré comme un luxe, la plupart des assurances ont collaboré à ce projet et admis qu'il pouvait y avoir un aspect économique au service rendu: L'économie est non seulement financière puisque les frais de transport et le recours à du personnel accompagnant sont épargnés mais aussi temporelle puisque la plupart des examens sont effectuées et interprétés dans la journée.
Nécessité d'une réadaptation du tarif TARMED

Après un soutien initial du projet par l'office fédéral de la santé, le projet a été avalisé avec une autorisation d'une durée de dix ans. Les départements de la santé des Cantons de Bâle-Ville, Bâle-Campagne et Genève ainsi que les représentants de Santé Suisse ont accepté de tenter l'expérience, étant entendu que l'utilité et l'économie apportée par ce concept devait faire ses preuves. Après trois ans, la preuve est faite mais la pérennité de la radiologie ambulatoire est menacée par l'omission d'une position cruciale dans TARMED: le transport du matériel diagnostique et du technicien en radiologie au lieu de résidence du patient n'ont pas été prévus. La demande est en cours. La réadaptation du tarif TARMED pour faciliter la prise en charge ambulatoire des patients est reconnue comme une nécessité à moyen et long terme (6).

Tenant compte de cette expérience positive nous sommes convaincus de l'utilité d'une radiologie ambulatoire qui permette la réduction des frais de transport et de personnel, offrant une contribution réaliste à la réduction du coût de la santé notamment en radiologie.

Nous pouvons mettre les atouts de la technologie moderne à disposition de nos personnes âgées et à mobilité réduite. Notre intention première, en tant que médecins, est d'accroître le confort de la personne handicapée et de ne pas négliger, pour raison d'un défaut d'infrastructure, le bien-être de la personne âgée que nous sommes tous appelés à devenir.

\section{Références}

1 Laerum F. Mobile X-ray room service rolls into Oslo's nursing homes. Diagnostic Imaging Supplmt. 2005.

2 Jeder zwölfte Deutsche ist schwerbehindert. Bull Méd Suisses. 2006;87(34):1463.

3 Baschung P, Stuck EE, Busato A. Die Verfügbarkeit ambulanter ärztlicher Behandlungen und deren Inanspruchnahme durch über 65-Jährige in der Schweiz. Bull Méd Suisses. 2008;89(6):233.

4 Burckhardt B, Nidecker A. Mobile Radiographie ein Versuch in der Region Nordwestschweiz und in den Kantonen Genf und Freiburg. Synapse. 2007(6).

5 Das Röntgen leicht gemacht: Altenpflege: Ein mobiles Röntgengerät soll älteren Patienten den Weg ins Spital ersparen. Basler Zeitung vom 23. Februar 2007.

6 Gähler E. Que vaut encore la médecine ambulatoire? Bull Méd Suisses. 2009;90(21/22):841. 